\title{
Discriminator and Diagnostic Features for Choroidal Malignant Melanoma and Choroidal Nevus
}

\author{
Burak Turgut, ${ }^{1}$ Onur Çatak, ${ }^{2}$ and Tamer Demir ${ }^{3}$
}

1. Faculty of Medicine, Department of Ophthalmology, Yuksek Ihtisas University, Ankara, Turkey; 2. Faculty of Medicine, Department of Ophthalmology, FIrat University, Elazig, Turkey; 3. Faculty of Medicine, Department of Ophthalmology, Onsekiz Mart University, Çanakkale, Turkey horoidal nevus and choroidal malignant melanoma, especially when they are small in size, may have similar ophthalmoscopic appearance. Additionally, though rarely, choroidal nevi may convert to malignant melanomas over several years. Early detection of a small choroidal malignant melanomas presents the risk of growth, and metastasis is extremely critical for the preservation of both vision and eye. Currently, the usage of the mnemonic TFSOM-HHD has been suggested to find small ocular melanomas using helpful hints daily. In this review, we aim to provide the main discriminator and diagnostic features for choroidal malignant melanoma and choroidal nevus.

\section{Keywords}

Choroidal, malignant melanoma, nevus, distinguishing, discrimination, clinical, imaging, mnemonic

Disclosure: Burak Turgut, Onur Çatak, and Tamer Demir have nothing to declare in relation to this article.

Review Process: Double-blind peer review.

Compliance with Ethics: This study involves a review of the literature and did not involve any studies with human or animal subjects performed by any of the authors.

Authorship: All named authors meet the International Committee of Medical Journal Editors (ICMJE) criteria for authorship of this manuscript, take responsibility for the integrity of the work as a whole, and have given final approval to the version to be published.

open Access: This article is published under the Creative Commons Attribution Noncommercial License, which permits any non-commercial use, distribution, adaptation and reproduction provided the original author(s) and source are given appropriate credit. (C) The Authors 2018.

Received: February 17, 2018

Accepted: March 16, 2018

Citation: US Ophthalmic Review. 2018;11(1):47-50

Corresponding Author: Burak Turgut, Professor of Ophthalmology, Yuksek Intisas University, Faculty of Medicine, Department of Ophthalmology, 06520, Ankara, Turkey. E: burakturgut@yiu.edu.tr

Support: No funding was received in the publication of this article.
Choroidal malignant melanoma (CMM) is the most common primary malignant intraocular tumor in adults. Significant risk factors for CMM include eyes with light-colored irises, such as green, blue, or gray; fair skin, cutaneous nevus and iris nevus, Caucasian ethnicity, and chronic sunlight exposure. CMMs often have: undefined boundaries; sharp elevation of the edges; an irregular, oblong configuration; association with subretinal fluid; and the inclusion of orange pigment.1-4

Choroidal nevi are usually gray-silver in color, have clear boundaries, and a flat or slightly elevated configuration. Choroidal nevi are usually asymptomatic and remain stable in size. However, drusen over nevus, atrophy, hyperplasia, or fibrous metaplasia in retinal pigment epithelium (RPE) may develop over time..$^{5}$ Choroidal nevi rarely convert to CMM, with a ten-year rate of 1 in 500 and an annual rate of 1 in 8,845. The rate of malignant transformation in a choroidal nevus is shown to increase with age. In other words, a choroidal nevus carries a small risk of less than $1 \%$ for malignant transformations. ${ }^{6-11} \mathrm{CMMs}$ tend to grow relatively rapidly, while as choroidal nevi, they may show slow enlargement over a period of several years. Although CMM can correctly be diagnosed with indirect ophthalmoscopy in over $95 \%$ of cases, ${ }^{12}$ it has been reported that approximately $3 \%$ of all choroidal nevi and $10 \%$ of "suspicious" choroidal nevi convert into CMM in 5 years. ${ }^{6-13} \mathrm{~A}$ choroidal nevus is defined by the Collaborative Ocular Melanoma Study (COMS) Group as any melanocytic choroidal lesion with less than $5 \mathrm{~mm}$ in the largest basal dimension and less than $1 \mathrm{~mm}$ in apical height. Giant nevus is defined as a nevus having a basal dimension, which is equal or more than $10 \mathrm{~mm}$, and it carries a greater risk for malignant transformation. A small CMM is defined as a CMM of $3 \mathrm{~mm}$ or less in apical height, with the largest basal diameter of 5-16 mm.6-13 It can be challenging to distinguish between the CMM from a choroidal nevus, especially in cases with a small CMM. To date, various risk factors for CMM have been reported in several studies. In the past, the risk factors for growth and possible malignant transformation, reported by COMS Group, were: greater initial tumor thickness (greater than $2 \mathrm{~mm}$ ) and diameter (greater than $7 \mathrm{~mm}$ ), the presence of orange pigment, the absence of drusen, and the absence of areas of RPE changes adjacent to the tumor. ${ }^{13}$ On the other hand, it has been considered that large or diffuse CMM (defined as a tumor with thickness $<20 \%$ of the tumor base with more horizontal tumor growth) is associated with poor prognosis and higher metastatic possibility. Shields et al. demonstrated that patients with small CMM of $3 \mathrm{~mm}$ or less in thickness on diffuse configuration had a greater risk of metastasis and death. ${ }^{14,15}$ The aim of this review is to provide the main discriminator and diagnostic features for CMM and choroidal nevus. 


\section{Mnemonic TFSOM-HHD}

Recently, the use of the mnemonic TFSOM-HHD has been suggested. This is a modified version of the former mnemonic, TFSOM-To Find Small Ocular Melanoma. The new mnemonic is To Find Small Ocular Melanoma using Helpful Hints Daily.9,16,17 The early detection of a small CMM carrying the risk for growth and metastasis is extremely critical for the preservation of both vision and eyeball. The new mnemonic modified by Shields et al. ${ }^{9}$ includes the following:

- Thickness greater than $2 \mathrm{~mm}$; this can be measured by optical coherence tomography (OCT) and fundus photography.

- Fluid in subretinal space; this is best revealed by OCT.

- symptoms.

- Orange pigment (lipofuscin) present; this can be best revealed by fundus autofluorescence (FAF).

- Margin within $3 \mathrm{~mm}$ of the disc; this can be measured by OCT and fundus photography.

- Hollowness in ocular ultrasonography (USG).

- Halo absent around lesion in fundus photography.

- Drusen absent on lesion; this can be observed with fundus photography and OCT.

Shield et al. reported a combination of factors, including symptoms such as flashing and floaters, the presence of orange pigment, proximity to the optic disc, ultrasonographic hollowness, and the absence of halo, which contributed to the highest hazard ratio for the growth of choroidal nevus into CMM.9.14,15 In contrast, singularly, the growth or diameter of the choroidal nevus was not considered as a precise diagnostic indicator of a CMM in the report. Studies on TFSOM-HHD risk factors have shown that a choroidal melanocytic lesion with none of the risk factors in the mnemonic presented only a $3 \%$ chance of growth over a five-year period. This lesion may most likely be considered a choroidal nevus. Lesions having just one factor have a 38\% chance of growth and should be observed. However, melanocytic lesions having three or more factors have a $50 \%$ greater chance of growth. ${ }^{14-18}$ Patients with at least three risk factors should be referred to an experienced ocular oncologist for possible CMM indications.

Currently, various imaging modalities are used for diagnosis of, and distinguishing between, CMM and choroidal nevus. The main findings from various imaging methods for CMM and choroidal nevus are given in Table 1.

\section{Fundus fluorescein angiography in CMM and choroidal nevus}

The differential diagnostic value of fundus fluorescein angiography (FFA) is lower because many choroidal nevi and small CMM have similar FFA findings, and FFA has no ability to provide detailed imaging of the choroidal circulation. There is no pathognomonic pattern of FFA for small CMM. However, FFA of CMM often reveals irregular and patchy fluorescence, including hypo-fluorescence due to masking by lipofuscin deposition in RPE and hyper-fluorescence due to window defect caused by RPE destruction in the early phase and the staining of the tumor or leakage in the late phase. Additionally, a pattern of "intrinsic tumor vasculature (double circulation)" and multiple central pinpoint leaks (hot spots) in the arteriovenous phase may be observed in the eyes with a mushroom-shaped or larger CMM. FFA of the choroidal nevus usually shows background hypo-fluorescence in an area corresponding to the nevus, or sometimes the scattered hyper- and hypo-fluorescent spots and small hyper-fluorescent patches corresponding to drusen. ${ }^{1,2,19,20}$

\section{Indocyanine green angiography in CMM and choroidal nevus}

Indocyanine green angiography (ICGA) findings in CMM may vary depending on the tumor pigmentation level, tumor thickness, and vascularity. However, ICGA often demonstrates hypo-fluorescence in the early phases, and sometimes intrinsic choroidal vasculature in the cases with CMM. In the late phase of ICGA, a hyper-fluorescence pattern or "washout" phenomenon may also be observed. The intrinsic choroidal vasculature (double circulation) is best visualized with ICGA. The "washout" phenomenon is defined as progressive choroidal hyper-fluorescence in small-caliber vessels in the initial phases, and complete obscuration of this hyper-fluorescence in the later phases. ${ }^{20,21}$ ICGA of choroidal nevi usually reveals a hypo-fluorescent region corresponding to the choroidal nevus until the late phase and sometimes scattered hyper- and hypo-fluorescent spots in the phases after the early phase. ${ }^{22}$

\section{Optical coherence tomography angiography in CMM and choroidal nevus}

OCT angiography (OCTA) is a new and noninvasive angiography type, which is used in the diagnosis of choroidal and retinal vascular diseases in the macula. In several recent studies, it has been reported that the OCTA images from choroidal nevi were mainly heterogenic and hyper-reflective, while the images of CMM were mainly iso-reflective or hypo-reflective. Additionally, the presence of a hypo-reflective plexus within the tumor and a hyper-reflective ring (in the choriocapillaris layer) surrounding the lesion were considered as a higher risk factor for malignancy. ${ }^{23}$ Additionally, it has been reported that eyes with macular CMM showed enlarged deep foveal avascular zone (FAZ) measurement, increased central macular thickness, and reduced superficial and deep capillary vascular density (CVD). In some eyes with choroidal nevus presenting three or more risk factors for growth, it has been found to show reduced deep CVD (dCVD). In this study, the reduction in superficial CVD is related to the presence of sub-retinal fluid. Additionally, this study demonstrated that dCVD reduction rate in eyes with nevus, versus those with CMM was approximately 1 in $6 .{ }^{24}$ In another study, OCTA of patients with choroidal nevus revealed a hypo-reflective mass with an intact RPE-Bruch's membrane complex, without marked deformity of choroidal vasculature. On the other hand, OCTA of the patients with CMM showed mural and piercing vessels and subtle changes in the RPEBruch's membrane complex, the growing tendency with flattening of the choriocapillaris, obscuration in RPE-Bruch's membrane complex and outer retinal layer. OCTA images have shown more axial or peripheral feeding vessels in choroidal melanomas than nevi. Additionally, choroidal vascular density (surface microvasculature) and flow in cases of CMM was found to be compared less to those with the nevus. In the nevi, the flow void mass was surrounded by a vascular rim. The CMM had more dilated and tortuous axial and peripheral feeding vessels as compared to nevi. ${ }^{25}$

\section{Fundus auto-fluorescence in CMM and choroidal nevus}

Lipofuscin is produced from the phagocytosis of the photoreceptor outer segments by RPE, which accumulates if RPE cannot perform this function. The excess accumulation in RPE results in further RPE dysfunction. Autofluorescence is caused by the lipofuscin. Thus, FAF allows us to imagine RPE changes and dysfunction. In FAF of a normal retina, the posterior pole shows a diffuse gray appearance due to mildly hyperautofluorescence caused by a signal from normal lipofuscin content in RPE cells in this region. The optic disc has a dark appearance due to the 


\begin{tabular}{|c|c|c|}
\hline Modality & CMM & Choroidal Nevus \\
\hline FFA & $\begin{array}{l}\text { Hypo-fluorescence due to lipofuscin deposition and hyper-fluorescence } \\
\text { due to RPE window defect in the early phase, the staining of the tumor or } \\
\text { leakage in the late phase, ICTV (double circulation) pattern and multiple } \\
\text { central pinpoint leaks in arteriovenous phase in the eyes with a mushroom- } \\
\text { shaped or larger CMM }\end{array}$ & $\begin{array}{l}\text { Moderate background hypo-fluorescence corresponding to nevus, } \\
\text { sometimes the scattered hyper- and hypo-fluorescent spots and small } \\
\text { hyper-fluorescent patches corresponding to drusen }\end{array}$ \\
\hline ICGA & $\begin{array}{l}\text { Hypo-fluorescence in early phases, sometimes ICTV, hyper-fluorescence } \\
\text { pattern or "washout" phenomenon in the late phase }\end{array}$ & $\begin{array}{l}\text { Moderate hypo-fluorescence corresponding to nevus until late phase, } \\
\text { sometimes scattered hyper- and hypo-fluorescent spots in later phases }\end{array}$ \\
\hline OCTA & $\begin{array}{l}\text { Iso-reflective/hyporeflective plexus within the tumor, a hyperreflective ring } \\
\text { surrounding the lesion, enlarged deep foveal avascular zone, increased } \\
\text { central macular thickness, reduced capillary vascular density }\end{array}$ & $\begin{array}{l}\text { Heterogenic and hyperreflective, hyporeflective mass with an intact } \\
\text { RPE-Bruch's membrane complex, flow void mass surrounded by a } \\
\text { vascular rim }\end{array}$ \\
\hline OCT & $\begin{array}{l}\text { Smooth, dome-shaped surface, choroidal shadowing, subretinal/intraretinal } \\
\text { fluid, shaggy photoreceptors or elongation or loss of photoreceptors, } \\
\text { choriocapillaris thinning, loss of ELM and IS-OS junction, irregularities in inner } \\
\text { plexiform layer and ganglion cell layer }\end{array}$ & $\begin{array}{l}\text { Smooth, mild dome-shaped surface, choroidal shadowing, choriocapillaris } \\
\text { thinning, photoreceptor attenuation/loss; RPE atrophy/loss/nodularity, IS- } \\
\text { OS junction irregularity/loss, irregularities in ELM, outer nuclear and outer } \\
\text { plexiform layers, inner nuclear layer }\end{array}$ \\
\hline FAF & $\begin{array}{l}\text { Patchy or diffuse FAF patterns, mild (in small CMM) or dense (in large } \\
\text { CMM) hyper-autofluorescence, slight intrinsic autofluorescence, a marked } \\
\text { extrinsic hyper-autofluorescence, confluent/clumped or speckled hyper- } \\
\text { autofluorescence pattern }\end{array}$ & $\begin{array}{l}\text { Patchy FAF pattern, slight intrinsic autofluorescence, hypo-autofluorescence } \\
\text { due to RPE hyperplasia/hypertrophy/atrophy, mildly hyper-autofluorescence } \\
\text { due to drusen }\end{array}$ \\
\hline A-USG & $\begin{array}{l}\text { Acoustic hollowness with low/medium internal reflectivity, highly reflective } \\
\text { anterior border, smooth sound attenuation, spontaneous vascular pulsations } \\
\text { in CMM }\end{array}$ & No significant finding \\
\hline B-USG & $\begin{array}{l}\text { Acoustic hollowness and orbital shadowing, smooth dome-shaped lesion } \\
\text { with low/medium internal echogenicity in CMM, choroidal excavation, } \\
\text { extrascleral extension, exudative retinal detachment, internal vascularity, } \\
\text { collar button or mushroom-shaped mass lesion }\end{array}$ & $\begin{array}{l}\text { Minimally vascular, flat, dome-shaped lesion, uniform medium/high internal } \\
\text { echogenicity, no internal vascularity; regular internal structure in the } \\
\text { posterior wall }\end{array}$ \\
\hline
\end{tabular}

A-USG = A scan ultrasonography; B-USG = B scan ultrasonography; $C M M=$ choroidal malignant melanoma; ELM = external limiting membrane; FAF = fundus auto-fluorescence; FFA = fundus fluorescein angiography; ICGA = indocyanine green angiography; ICTV = intrinsic choroidal tumor vasCulature; IS-OS = inner segment-outer segment; OCT = optical coherence tomography; OCTA = OCT angiography; RPE = retina pigment epithelium.

absence of RPE. All vasculature in the retina and the fovea also seem dark due to absorption from blood or macular lutein, respectively. Lipofuscin is a risk factor for CMM. Thus, FAF may be helpful in the discrimination of CMM from a choroidal nevus. In literature, the FAF patterns have been defined as patchy or diffused in choroidal melanoma, and patchy in choroidal nevi. The patchy pattern is characterized by the presence of distinct areas of increased FAF between areas of normal autofluorescence. The diffuse pattern is characterized by the presence of increased FAF with indistinct borders over a larger part ( $>50 \%)$ of the tumor in the absence of the intervening areas. ${ }^{26-31}$

Choroidal nevi demonstrate only the patchy pattern and little intrinsic autofluorescence. They often have a darkly hypo-autofluorescent appearance from overlying RPE hyperplasia/hypertrophy and atrophy. Drusen, which usually exist in choroidal nevi, typically appears in a mild hyper-autofluorescence pattern. Lipofuscin, which may be present in onethird of cases with choroidal nevi has the brightest hyper-autofluorescence. Subretinal fluid, which is more indicative of CMM, may exists in one-sixth of cases with choroidal nevus usually displaying mild hyper-fluorescence. ${ }^{26-31}$

CMMs are presented with a diffused or patchy pattern. CMMs often show slight intrinsic autofluorescence from fluorophores in subretinal fluid and a marked extrinsic hyper-autofluorescence from overlying lipofuscin within RPE. Hyper-autofluorescence pattern is in a form of confluent/clumped or speckled hyper-autofluorescence. ${ }^{26-31}$

\section{Optical coherence tomography in CMM and choroidal nevus}

Specifically, swept source OCT (SS-OCT) or enhanced depth imaging (EDIOCT) allow more precise detection of the exact location, dimensions, depth, and demarcation boundaries of small and larger amelanotic CMMs or choroidal nevus from the surrounding healthy tissue. However, visualization of the deeper layers of the choroid and sclera with OCT may be limited by light scattering from pigmentary tissue and minimal penetration into the choroidal mass. ${ }^{32-35}$

Characteristic OCT findings of a CMM include a smooth, dome-shaped surface, serous retinal detachment or subretinal/intraretinal fluid, subretinal lipofuscin deposition, intact but elongated/shaggy photoreceptors, choroidal shadowing, choriocapillaris thinning, normal retinal thickness, and increased tumor thickness. ${ }^{32-35}$

OCT findings of a choroidal nevus are often a lesion with smooth, mild dome-shaped surface and choroidal shadowing deep into the nevus, choriocapillaris thinning overlying the nevus, retinal thinning and attenuation or loss in photoreceptors, atrophy or loss, or nodularity in RPE, irregularity in outer retinal layers, inner segment-outer segment (IS-OS) junction irregularity/loss, and sometimes subretinal fluid. The hypo-reflectivity occurs in two out of three cases with pigmented choroidal nevus and one in five to one in six cases with nonpigmented ones. ${ }^{32-35}$ 
Compared with similar-sized choroidal nevi, distinctive OCT findings of CMM are intraretinal edema, elongation or loss of photoreceptors, loss of the external limiting membrane and inner-outer junction, and irregularity in the inner plexiform layer and ganglion cell layer. ${ }^{32-35}$

\section{$A$ and $B$ scan ultrasonography in CMM and choroidal nevus}

A-scan USG of patients with CMM may reveal acoustic hollowness with medium-to-low internal reflectivity, a highly reflective anterior border of the CMM, smooth sound attenuation, and spontaneous vascular pulsations within the tumor. However, A-scan cannot provide significant findings in choroidal nevus. ${ }^{1,2,18,19,36}$

B-Scan USG reveals that the classic triad of CMM is an acoustically hollow zone within the melanoma, choroidal excavation, and orbital shadowing in the orbit. Additionally, it may reveal low-to-medium internal reflective echoes, extra-scleral tumor extension, exudative retinal detachment, internal vascularity, a collar button, or mushroom-shaped mass lesion. A combination of A- and B-scan USG allows us to diagnose CMMs with greater than $95 \%$ accuracy in tumors greater than $3 \mathrm{~mm}$ in thickness. Choroidal nevi are seen as flat, minimally vascular, medium, or high echogenic lesions with a regular internal structure in the posterior wall in ocular USG. However, the thin nature of nevi causes difficulty in their differentiation from small CMM. ${ }^{12,18,19,36}$

\section{Conclusion}

Recently, the use of the mnemonic TFSOM-HHD has been suggested as a way to find small ocular melanomas using helpful hints daily. The use of this mnemonic and multimodal imaging modalities will provide practicality in the diagnosis, evaluation for malignant transformation, and differential diagnosis of CMM and choroidal nevus. $\square$
1. Singh P, Singh A. Choroidal melanoma. Oman J Ophthalmol. 2012:5:3-9.

2. Augsburger JJ, Damato BE, Bornfeld N, Uveal Melanoma. In Yanoff M, Duker JS (eds). Ophthalmology. First Edition, London: Mosby, 1999; 1052-63.

3. Lederer DE, Edelstein C. Choroidal melanoma: clinical presentation and differential diagnosis. Can J Ophthalmol. 2004;39:358-64.

4. Shah $\mathrm{CP}$, Weis $\mathrm{E}$, Lajous $\mathrm{M}$, et al. Intermittent and chronic ultraviolet light exposure and uveal melanoma: a meta-analysis. Ophthalmology. 2005;112:1599-607.

5. Chien JL, Sioufi K, Surakiatchanukul T, et al. Choroidal nevus: a review of prevalence, features, genetics, risks, and outcomes Curr Opin Ophthalmol. 2017;28:228-37.

6. Singh AD, Kalyani P, Topham A. Estimating the risk of malignant transformation of a choroidal nevus. Ophthalmology. 2005;112:1784-9

7. Kaiserman I, Kaiserman N, Pe'er J. Long term ultrasonic follow up of choroidal naevi and their transformation to melanomas. $\mathrm{Br} J$ ophthalmol. 2006;90:994-8.

8. Gass JD. Problems in the differential diagnosis of choroidal nevi and malignant melanomas, The XXXIII Edward Jackson Memoria Lecture. Am J Ophthalmol. 1977;83:299-323.

9. Shields $\mathrm{CL}$, Furuta M, Berman EL, et al. Choroidal nevus transformation into melanoma: Analysis of 2514 consecutive cases Arch Ophthalmol. 2009:127:981-7.

10. Augsburger JJ, Correa ZM, Trichopoulos N, Shaikh A. Size overlap between benign melanocytic choroidal nevi and choroidal malignant melanomas. Invest Ophthalmol Vis Sci. 2008;49:2823-8.

11. Mashayekhi A, Siu S, Shields CL, Shields JA. Slow enlargement of choroidal nevi: a long-term follow-up study. Ophthalmology. 2011;118:382-8.

12. Char DH, Stone RD, Irvine AR, et al. Diagnostic modalities in choroidal melanoma. Am J Ophthalmol. 1980;89:223-30.

13. The Collaborative Ocular Melanoma Study Group. Factors predictive of growth and treatment of small choroidal melanoma:
COMS report no 5. Arch Ophthalmol 1997:115:1537-44.

14. Shields $\mathrm{CL}$, Shields JA, Kiratli $\mathrm{H}$, De Potter P, Cater JR, Risk factors for growth and metastasis of small choroidal melanocytic lesions. Ophthalmology. 1995;102:1351-61.

15. Shields $\mathrm{CL}$, Cater J, Shields JA, et al. Combination of clinical factors predictive of growth of small choroidal melanocytic tumors. Arch Ophthalmol. 2000;118:360-4.

16. Rishi P, Koundanya WV, Shields CL. Using risk factors for detection and prognostication of uveal melanoma. Indian J Ophthalmol. 2015:63:110-6.

17. Shields $C L$. The hunt for the secrets of uveal melanoma. Clin Exp Ophthalmol. 2008;36:277-80.

18. Kaliki S, Shields $C L$, Shields JA. Uveal melanoma: Estimating prognosis. Indian J Ophthalmol. 2015;63:93-102.

19. Bakri SJ, Sculley L, Singh AD. Diagnostic techniques. In Singh AD (ed). Clinical Ophthalmic Oncology. First Edition, Edinburgh: Elsevier-Saunders, 2007: 175-80.

20. Atmaca LS, Batioğlu F, Atmaca P. Fluorescein and indocyanine green videoangiography of choroidal melanomas. Jpn J Ophthalmol. 1999;43:25-30.

21. Shields $C L$, Shields JA, De Potter P. Patterns of indocyanine green videoangiography of choroidal tumours. Br J Ophthalmol. 1995;79:237-45

22. Shiraki $\mathrm{K}$, Moriwaki $\mathrm{M}$, Yanagihara $\mathrm{N}$, et al. Indocyanine green angiograms of choroidal nevi: Comparison between confocal and nonconfocal scanning laser ophthalmoscope and fundus video camera. Jpn I Ophthalmol 2001:45:368-74.

23. Toledo J, Asencio M, García JR, et al. OCT Angiography: Imaging of choroidal and retinal Tumors. Ophthalmology Retina. 2017; in press. DOl: https://doi.org/10.1016/j.oret.2017.10.006

24. Valverde-Megías A, Say EA, Ferenczy SR, Shields CL. Differential macular features on optical coherence tomography angiography in eyes with choroidal nevus and melanoma. Retina. 2017:37:731-40.

25. Ghassemi F, Reza Mirshahi R, Fadakar K, Sabour S. Optical coherence tomography angiography in choroidal melanoma and nevus. Clin Ophthalmol 2018:12:207-14.

26. Gunduz K, Pulido IS, Ezzat K, et al. Review of fundus autofluorescence in choroidal melanocytic lesions. Eye (Lond). 2009;23:497-503.

27. Lavinsky D, Belfort RN, Navajas E, et al. Fundus autofluorescence of choroidal nevus and melanoma. Br J Ophthalmol. 2007:91:1299-302.

28. Shields $C L$, Bianciotto $C$, Pirondini $C$, et al. Autofluorescence of choroidal melanoma in 51 cases. Br J Ophthalmol. 2008:92:61722.

29. Amselem L, Pulido JS, Gunduz K, et al. Changes in fundus autofluorescence of choroidal melanomas following treatment. Eye (Lond). 2009;23:428-34.

30. Chin K, Finger PT. Autofluorescence characteristics of suspicious choroidal nevi. Optometry. 2009;80:126-30.

31. Materin MA, Raducu R, Bianciotto C, Shields CL. Fundus autofluorescence and optical coherence tomography findings in choroidal melanocytic lesions. Middle East Afr $J$ Ophthalmol. 2010;17:201-6.

32. Medina CA, Plesec T, Singh AD. Optical coherence tomography imaging of ocular and periocular tumours. Br J Ophthalmol. 2014;98:40-6.

33. Shah SU, Kaliki S, Shields $\mathrm{CL}$, et al. Enhanced depth imaging optical coherence tomography of choroidal nevus in 104 cases Ophthalmology. 2012:119:1066-72.

34. Shields CL, Kaliki S, Rojanaporn D, et al. Enhanced depth imaging optical coherence tomography of small choroidal melanoma: comparison with choroidal nevus. Arch Ophthalmol. 2012;130:850-6

35. Shields CL, Mashayekhi A, Materin MA, et al. Optical coherence tomography of choroidal nevus in 120 patients. Retina. 2005;25:243-52

36. De La Hoz Polo M, Torramilans Lluís A, Pozuelo Segura O, et al. Ocular ultrasonography focused on the posterior eye segment: what radiologists should know. Insights Imaging. 2016;7:351-64. 